\title{
Bibliographical analysis of research on smoking cessation therapy
}

\author{
John R Hughes, Anthony Liguori
}

\begin{abstract}
Objective-To compare the growth of research on treatments for smoking with that for similar medical/behavioural disorders.

Design-We surveyed Medline for 19671994 for articles on smoking, alcohol, anxiety, and obesity disorders.

Main outcome measure-Number of articles published in two-year intervals.

Results-The number of articles per year on smoking/nicotine in humans increased fivefold from 1967 to 1994 compared with twofold for Medline as a whole. The rate of growth of empirical studies of treatment for smoking was as great as, or greater than, that for alcohol, anxiety, or obesity problems. In recent years, the rate of publication has continued to increase for drug treatments for smoking, has plateaued for brief advice, and has declined for behaviour therapy.

Conclusion-Research on smoking is increasing as much as, if not more than, research in several similar disorders. The one area of apparent decline in smoking research is behaviour therapy for smoking cessation.
\end{abstract}

(Tobacco Control 1997;6:111-114)

Keywords: bibliography, smoking cessation, tobacco use

\section{Introduction}

A comparison of the three reviews by Schwartz on the treatment of smoking ${ }^{1-3}$ suggests that the rate of publication of studies of treatments for smoking cessation has increased over the last few decades; however, others have noted a decline in the rate of publication in certain areas of treatment, such as behaviour therapy. ${ }^{4}$ The goal of our study was to determine whether research on treatments for smoking was growing and, if so, how such growth compared with the growth of treatment research in other fields. In addition, we sought to determine trends in publication of studies for different treatments. Specifically, the study used a computerised reference search to quantify the rate of publication and types of treatments studied over time, and used treatment for alcohol, anxiety disorders, and obesity as comparisons. ${ }^{5}$

\section{Methods}

We used Medline to find references on smoking, alcohol, obesity, and anxiety disorders from 1967 (the first full year of Medline listings) through 1994. Medline focuses on approximately 3800 medical journals. We attempted to search PSYCHLIT to include psychology journals; however, this database does not differentiate research vs opinion pieces, and animal vs human studies, does not use subheadings, and only began in 1983. For these reasons, we chose not to use PSYCHLIT, except for a limited search mentioned below.

Alcohol, anxiety, and obesity were chosen as controls, because each is a behavioural disorder and has a tradition of empirical studies of both behavioural and pharmacological treatments. We compiled the references in twoyear intervals, and limited our search to articles on humans. We used five focus descriptors for "smoking" (nicotine, smoking, smoking cessation, tobacco, and tobacco use disorder), 12 for "alcohol" (alcohol amnestic disorder, alcohol deterrents, alcohol drinking, alcohol withdrawal delirium, alcoholic beverages, alcoholic intoxication, alcoholic psychosis, alcoholics anonymous, alcoholism, fetal alcohol syndrome, skid row alcoholics, and temperance), five for "anxiety" (anxiety, anxiety disorders, castration anxiety, dental anxiety, and separation anxiety), and three for "obesity" (obesity in diabetes, obesity, and morbid obesity). These search terms were taken from the Medical Subjects Headings (MESH) in Index Medicus. ${ }^{6}$ Thirty randomly selected articles in each heading were reviewed to verify that the heading covered the type of articles intended.

We then did a second search limiting the focus to empirical studies using "clinical trial" as a publication type or using "comparative study", "double-blind method", or "follow-up studies" as focus descriptors. We further limited the empirical studies to drug therapy subgroups by adding the subheadings "drug therapy" or "therapeutic use" to the "alcohol", "anxiety", "obesity", and "smoking" terms. For "nicotine", we also used the subheading "administration and dosage" because nicotine replacement trials were described within this subheading.

We attempted to search for psychosocial therapies across smoking, obesity, and anxiety disorders with the subheadings "therapy" and "rehabilitation", but we found that these classifications appeared to be used differently across conditions. Because of this, we limited our search for psychosocial therapies only to nicotine/smoking. We focused on two treatments: brief therapy and behaviour therapy. Brief therapy was searched with the keywords "counselling", "programmed instruction", and "social support". Inspection of 

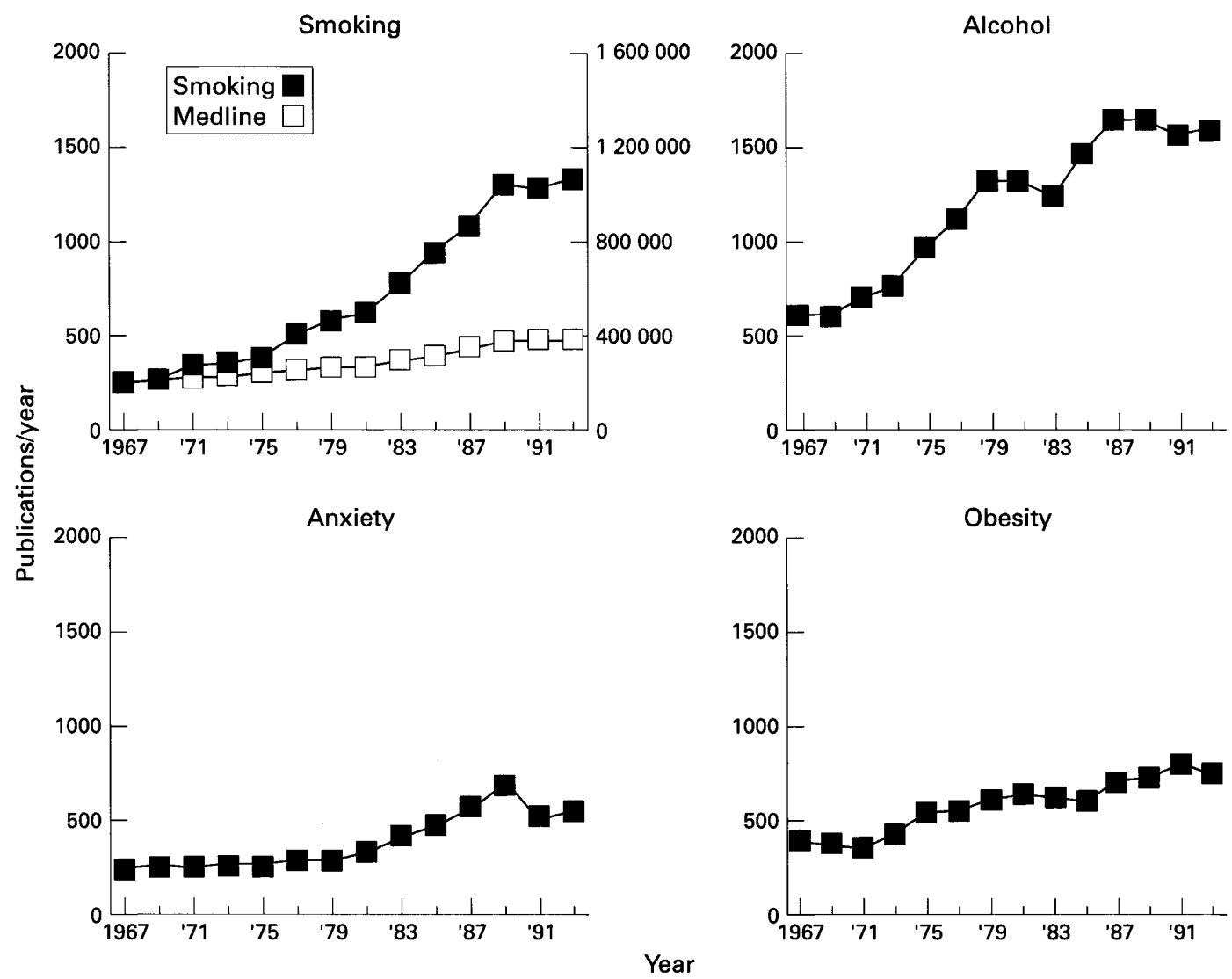

Figure 1 Each data point represents the mean number of publications per year for the two-year period beginning with the identified year. The left axis is for smoking; the right axis is for all of Medline.
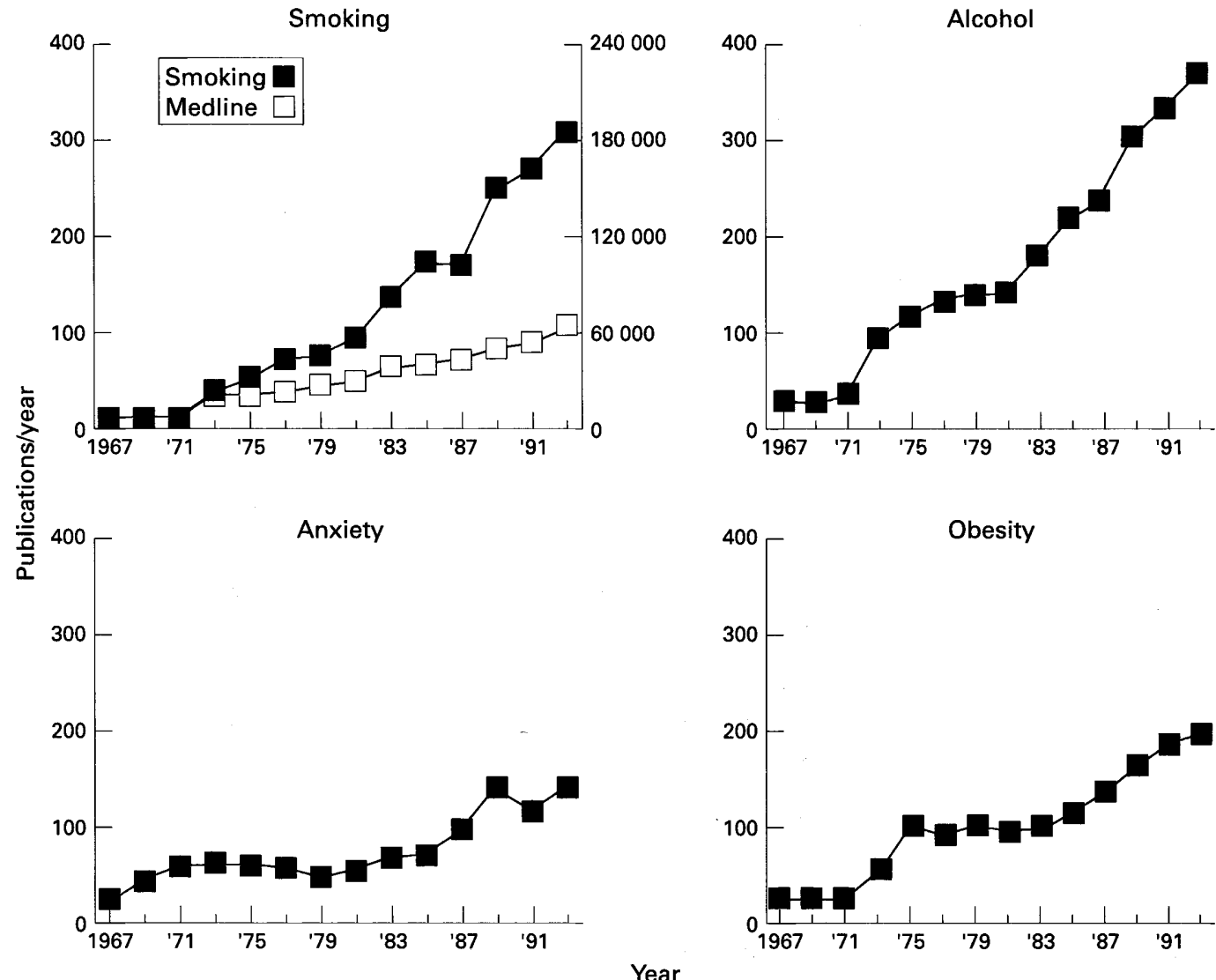

Figure 2 Same as figure 1, but for empirical studies only. See text for the operational definition of empirical. 

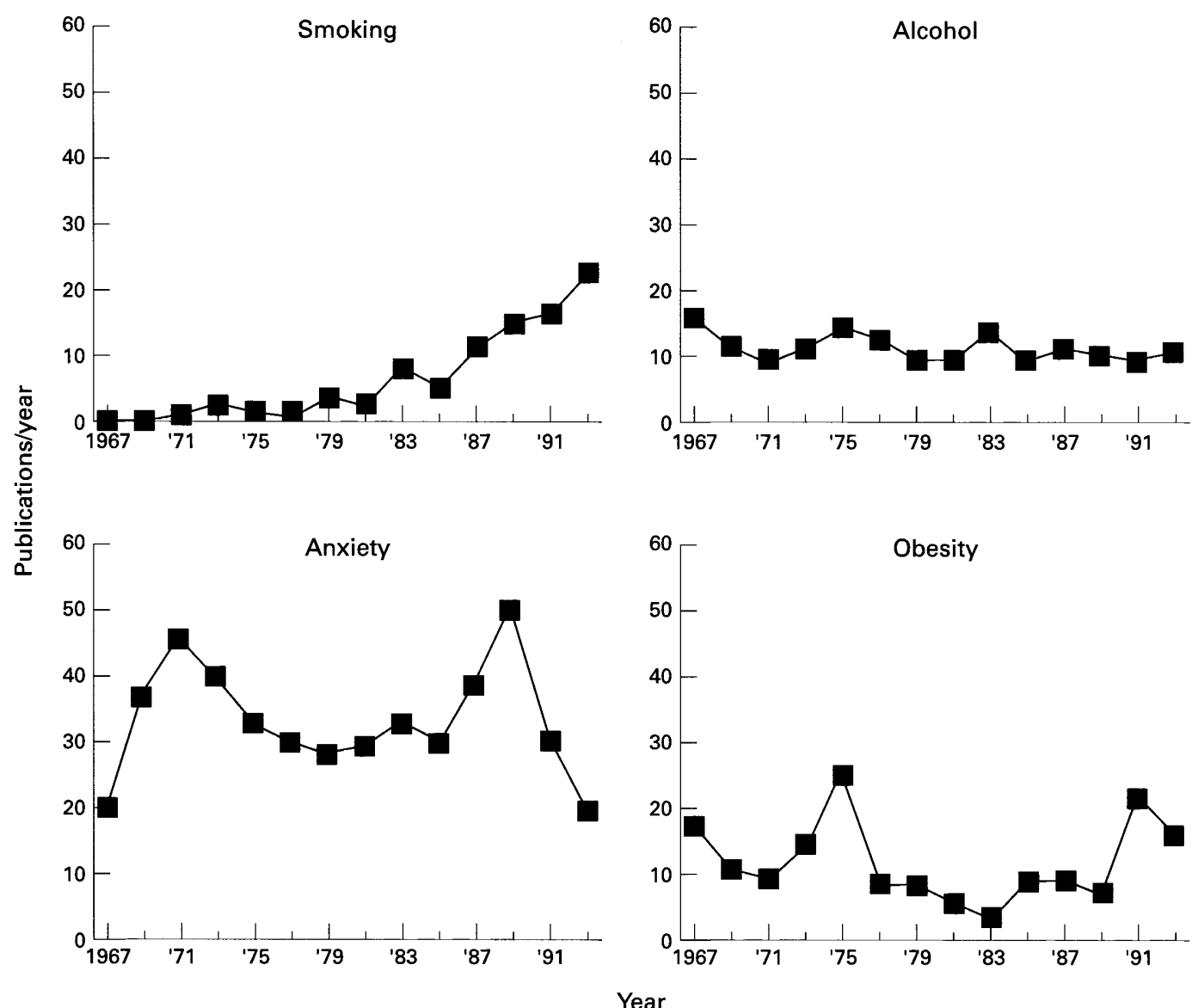

Figure 3 Same as figure 1, but for pharmacotherapy. See text for the operational definition of pharmacotherapy.

the outcome indicated that this produced selfhelp, physician advice, telephone interviewing, and other forms of minimal interventions. We searched for behaviour therapy with the keywords "behaviour therapy" or "cognitive therapy" foci. Finally, we determined the number of meta-analyses and review articles by limiting the search to "meta-analysis" or "review" as publication type.

\section{Results}

The total number of publications on nicotine/smoking began increasing in the 1970s and grew steadily until 1989 (figure 1). The rate of publication appears to have levelled off in the past four years. Since 1989, approximately 1300 articles on smoking/nicotine have appeared each year. This increase in smoking/nicotine articles from 1967 to 1994 (a fivefold increase) is substantially greater than that in Medline as a whole (a twofold increase). The rate of growth and number of publications for smoking/nicotine were similar to that for alcohol but greater than that for anxiety and obesity. Similar results were found when we searched PSYCHLIT for all articles on these topics.

When we examined only empirical studies, again the rate for smoking/nicotine was greater than that for Medline, and, in this case, no plateauing occurred (figure 2). The growth and number of empirical articles were again similar to that for alcohol but greater than that for obesity and anxiety disorders.
When we examined only drug therapies, we found the same increasing trend without plateauing for smoking studies but the increase began later, during the 1980 s (figure 3). In contrast, the numbers of pharmacotherapy studies on alcoholism, anxiety, and obesity have remained fairly constant.

The number of empirical studies of brief 3 therapies for smoking cessation increased in the 1980 s and climbed to $12-14$ per year but appears to have plateaued since 1989 (figure 윽 4). Studies of behaviour therapy for smoking cessation began to increase in the 1970 s and, except for the $1979-80$ period, rose steadily to 16-20 per year in 1987-90 but has fallen dramatically since then (figure 4). The number of $N$ meta-analysis and review articles showed an $\mathrm{\omega}$ increase until the late 1980 s and more recently a decline.

\section{Conclusions}

Research into smoking and treatments for $\frac{\vec{D}}{\bar{D}}$ smoking cessation has grown dramatically in $\frac{\rho}{8}$ the 1980s and early 1990s. This growth is $\varrho$ much greater than that seen in Medline as a whole and is equal to or greater than that seen 8 for alcohol, obesity, and anxiety disorders. However, there are exceptions: studies on behavioural/cognitive therapy for smoking $\stackrel{?}{\risingdotseq}$ appear to have declined recently and studies on brief therapies appear to have plateaued. Two possible reasons for the decline in behavioural studies include the lack of innovative treatments ${ }^{4}$ and the realisation of the problems 

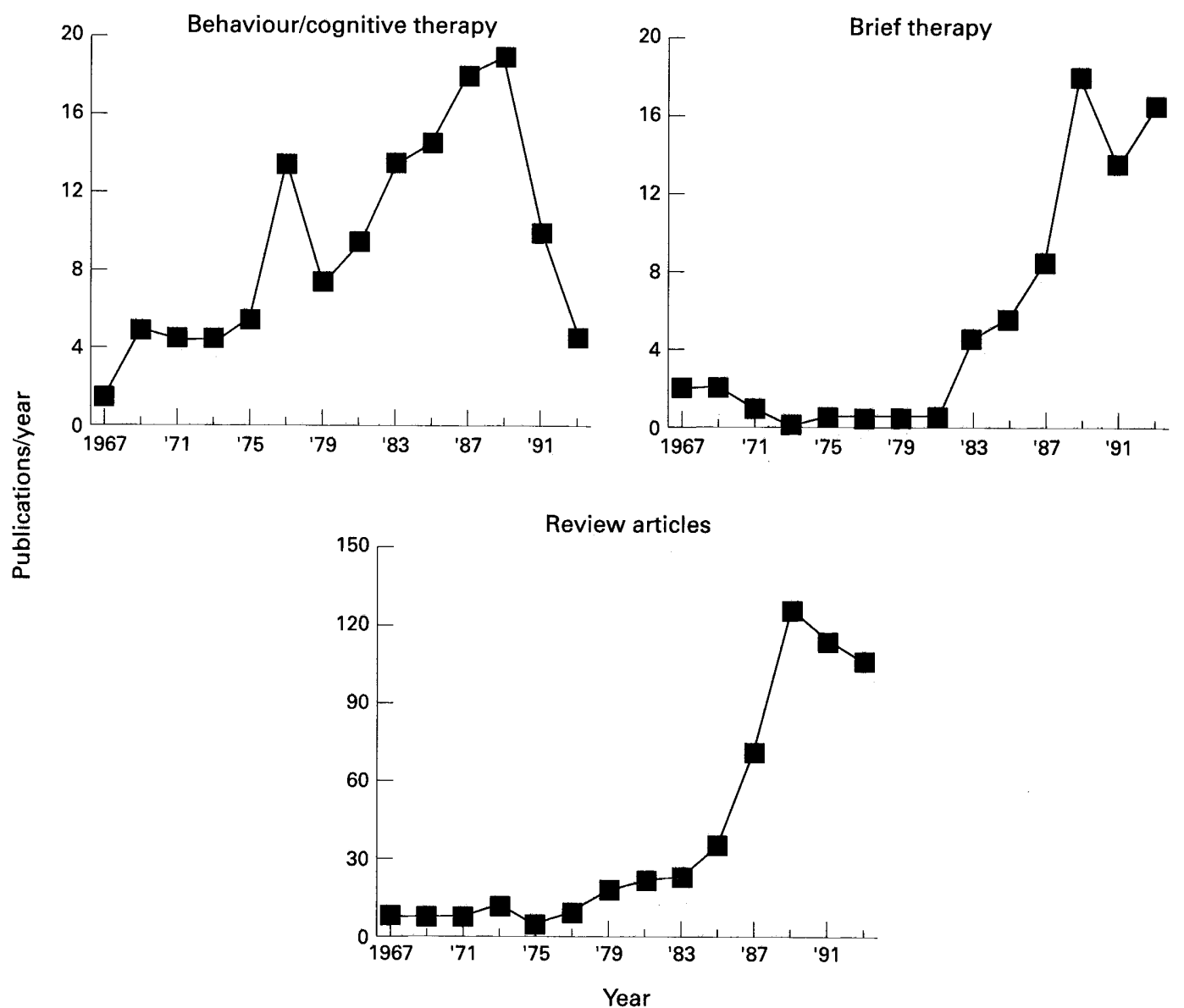

Figure 4 Same as figure 1, but for specific types of studies. See text for the operational definitions of the study types.

in making behaviour therapy available, accessible, and reimbursable. ${ }^{7}$ Two possible reasons for the plateau of interest in brief therapies include (a) the relatively low rates of success, ${ }^{8}$ and (b) recognition of the need for more intensive therapies as remaining smokers become those more dependent on nicotine or with more comorbidities. ${ }^{8}$

As in all studies using bibliographical and other databases, our study was limited by the characteristics of the database. ${ }^{5}$ First, Medline focuses on medical journals, not psychology journals. Second, for this period, several relevant journals were not indexed in Medline-for example, Tobacco Control (which is now included in Medline ${ }^{9}$ ). Third, indexing methods for behavioural disorders and for non-pharmacological treatments appear to be less reliable than for physical disorders and for pharmacotherapies. ${ }^{5}$ Fourth, due to abstracting methods, our study could not assess several treatments types-for example, mass media and non-nicotine medications. As a result of these limitations, readers should not overinterpret the accuracy of our actual numbers but should focus instead on trends over time.

In summary, despite the fact that journals and research societies on smoking/nicotine have only recently appeared, and despite the absence of a single academic home or funding institute, research on smoking treatment is growing to a degree comparable to or greater than that for other complex behavioural disor- ders. The importance of this research database is evidenced by the close agreement between research findings and accepted treatments for smoking cessation. For example, most of the recommendations of the two recent consensus guidelines for treating smoking are based on meta-analyses of research studies. ${ }^{10} 11$

This study was funded in part by Research Scientist Development Award DA-00109 (Dr Hughes) from the National Institute on Drug Abuse and grant AA-09480 from the National Institute on Alcohol Abuse and Alcoholism.

1 Schwartz JL. Methods of smoking cessation. Med Clin North Am 1992;76:451-76.

2 Schwartz JL. Review and evaluation of smoking cessation methods: United States and Canada, 1978-1985. Washington, DC: US Department of Health and Human Services, National Institutes of Health, 1987. (NIH Publication No 87-2940.)

3 Schwartz JL. A critical review of smoking control methods. Public Health Rep 1969;84:483-506.

4 Shiffman S. Smoking cessation treatment: Any progress? 7 Consult Clin Psychol 1993;61:718-22.

5 Hughes JR, Oliveto A. The growth of treatment research in drug and alcohol use disorders. Drug Alcohol Dependence 1990;26:81-4.

6 National Institutes of Health. Medical subject headings; supplement to Index Medicus. Bethesda, Maryland: National Institutes of Health, 1996.

7 Lichtenstein E, Glasgow RE. Smoking cessation: What have we learned over the past decade? $\mathcal{F}$ Consult Clin Psychol 1992;60:518-27

8 Foulds J. Strategies for smoking. Br Med Bull 1997. In press. Davis RM. Tobacco Control joins Index Medicus and Medline. Tobacco Control 1996;5:99.

10 Hughes JR, Fiester S, Goldstein MG, Resnick MP, Rock N, Ziedonis D. American Psychiatric Association Practice Guideline For the Treatment of Nicotine Dependence. Guideline For the Treatment of
Am $\mathcal{F}$ Psychiatry 1996;153:S1-31.

11 Smoking Cessation Clinical Practice Guideline Panel and Staff. The Agency for Health Care Policy and Research Smoking Cessation Clinical Practice Guideline. $\mathscr{F} A M A$ 1996;275:1270-80 